Test Method

\title{
Length scale effects in epoxy: The dependence of elastic moduli measurements on spherical indenter tip radius
}

\author{
Gurudutt Chandrashekar ${ }^{\star}$, Chung-Souk Han \\ University of Wyoming, Department of Mechanical Engineering, 1000 E. University Ave., \\ Laramie, WY 82071, USA
}

\begin{abstract}
Significant increases in the measured elastic moduli with decreasing indentation depth have been previously found in various polymers by indentation tests with a Berkovich tip at micro- to nanometer length scales. These increases in the determined elastic moduli were related to second order displacement gradients which increase with decreasing depth when a conical tip is applied. When a spherical tip is applied, such depth dependence should not be present as the second order displacement gradients remain essentially unchanged with indentation depth. However, these gradients should be proportional to the radius of the spherical tip. To examine the notion of second order displacement gradient dependence in measurements of elastic moduli, indentation experiments are conducted on epoxy with spherical tips of different nominal radii. Accounting for tip imperfections, an increase in the determined elastic moduli is found with decreasing tip radius, which corroborates the notion of second order displacement gradient dependence.
\end{abstract}

KEYWORDS: 20 moduli; spherical tip; tip radius; second order displacement gradients

- Corresponding author. Tel.: +1 307-761-1572

E-mail address: gchandra@uwyo.edu (Gurudutt Chandrashekar). 


\section{Introduction}

Indentation testing has been widely applied to characterize and determine properties of materials at nano- to micrometer length scales [1-12]. The deformation mechanisms and behavior at these length scales can be significantly different compared to those at the macroscopic length scale, and nanoindentation testing has been a vital tool to unravel these differences. Using nanoindentation, length scale dependent deformation has been experimentally observed in metals as well as in polymers. With respect to length scale dependent deformation in metals, significant experimental evidence is available in the literature. However, for polymers, experiments on length scale dependent deformation are comparatively scarce and the deformation mechanisms are far from clear.

Indentation testing with Berkovich tips revealed length scale dependent deformation in various polymers such as PDMS [6,7], natural rubber [8] and epoxy [9-11], where significant increases in hardness were observed with decreasing indentation depth, $h$. A recent comprehensive review of the length scale dependent deformation in polymers can be found in [13]. These increases in the hardness could be predicted by a non-local, second order displacement gradient dependent deformation [14-16]. Besides increases in hardness, increases in the measured elastic moduli, $E$, with decreasing $h$ were also found with Berkovich tips for various polymers $[7,8,10]$. In contrast to the pyramidal Berkovich tip, indentation testing on these polymers with a spherical tip of large curvature radius resulted in $E$ remaining essentially constant with $h[7,8,10]$. The different trends in measured $E$ with Berkovich and spherical tips can be explained with second order displacement gradients which increase with decreasing $h$ for a Berkovich tip but remain essentially constant with $h$ for a spherical tip. However, with a spherical tip, these gradients scale with the inverse of tip radius. To examine this notion, an 
indentation study is conducted here to determine the influence of spherical tip radius on the measurements of $E$.

Indentation with spherical tips has been widely applied to characterize the mechanical properties of metals, ceramics and polymers [7,8,10,11,17-29]. Indentation experiments with spherical tips have the advantages of gradual increases in stresses and elastic deformation dominating the initial stages of indentation testing. These lower stresses and elastic deformation enable the utilization of the Hertz contact theory [30] in the material characterization. With the onset of plastic deformation, however, the approach of Oliver and Pharr [31] is widely applied to determine mechanical properties such as hardness and elastic modulus.

The nominal tip radius of a spherical tip is usually specified by the manufacturer. But, as with Berkovich tips, imperfections in the geometry of a spherical tip will always be present and assuming the specified nominal tip radius as the actual value can lead to erroneous results $[17,19,21,27]$. Consequently, tip imperfections have to be examined and its influence on the indentation results studied, especially at smaller indentation depths where small imperfections can strongly influence the measurements.

Length scale dependent deformation is of growing importance with ever decreasing dimensions of components and material phases. The corresponding decreasing length scales in the material testing necessitates a clear understanding of the length scale dependent mechanisms. In the present work, epoxy is considered as a model polymer to investigate the dependence of the measured elastic moduli on the radius of the spherical tip. Compared to soft polymeric materials such as PDMS and natural rubber, indentation testing on the relatively stiff epoxy involves reduced difficulties with respect to surface detection, adhesion, etc $[7,8,10]$. In this study, the elastic moduli are determined at various indentation depths with spherical tips of 3,10,50 and 
$250 \mu \mathrm{m}$ nominal radii. To address tip imperfections, the tips are examined with fused silica as a reference material. The results of these experiments are discussed in view of other possible factors and interpretations.

\section{Experiments}

\subsection{Sample preparation}

Epoxy samples were prepared by mixing the commercially available liquid epoxy resin, EPON 828 (bisphenol A-epichlorohydrin), as base agent with the curing agent EPIKURE 3223, diethylenetriamine (DETA). The epoxy resin (Momentive Specialty Chemicals, USA) and DETA (Momentive Specialty Chemicals, USA) were used in the sample preparation following a procedure identical to that followed in [10], except that a curing agent content of $9 \mathrm{phr}$ (parts per hundred resin) was used in the sample preparation, as opposed to $20 \mathrm{phr}$.

\subsection{Indentation testing}

The indentation experiments were conducted at room temperature of about $23^{\circ} \mathrm{C}$ with spherical tips of 3, 10, 50 and $250 \mu \mathrm{m}$ nominal radii on an Agilent G200 nanoindenter system equipped with XP and DCM heads. The 3,10 and $50 \mu \mathrm{m}$ radii tips were purchased from SYNTON-MDP AG (Nidau, Switzerland) and the $250 \mu \mathrm{m}$ tip was purchased from Micro Star Tech (Huntsville, TX, USA). The $3 \mu \mathrm{m}$ radius tip was mounted on the DCM head and the other three tips on the XP head. The XP and DCM heads have load limits of $500 \mathrm{mN}$ and $30 \mathrm{mN}$, respectively, which correspondingly limit the maximum indentation depth. The indentation experiments were conducted in two stages:

1) Tip imperfection study using fused silica as the reference material.

2) Determination of elastic moduli of the epoxy sample. 
All indentation experiments were conducted at room temperature in force controlled mode with the loading and unloading being applied linearly in time. Fig. 1 depicts a typical loaddisplacement curve with loading (A-B), holding (B-C) and unloading (C-D) sequences. The figure also illustrates the maximum force $F_{\max }$, indentation depth $h$, maximum depth $h_{\max }$ (at $\left.F_{\text {max }}\right)$ and final depth $h_{\mathrm{f}}($ at $F=0)$.

In addition to the test with spherical tips, a Berkovich tip (which was mounted on the XP head) was applied to determine $E$ over $h$.

\subsubsection{Tip imperfection study}

As mentioned previously, a nominal tip radius, $R_{\text {nom }}$ is usually provided by the manufacturer. However, imperfections in the curvature have to be expected due to complexities in the manufacturing process. Tip imperfection studies conducted to assess the deviation of the spherical curvature from the nominal tip radius can be found in $[17,19,27]$. One such study performed by Constantinidis et al. [27] utilized two different techniques: a) Indentations on fused quartz and b) AFM imaging, to determine the tip imperfections. Based on the first technique, a study was conducted here on a standard reference sample - fused silica (elastic modulus $=72.1$ GPa and Poisson's ratio $=0.18)$ to assess the tip imperfections of the 3,10 and $50 \mu \mathrm{m}$ tips. A tip imperfection study on the $250 \mu \mathrm{m}$ tip was omitted for reasons to be discussed below.

Previously, using the Oliver and Pharr approach [31], Herbert et al. [18] determined the elastic moduli of fused silica and Aluminium 6061-T6 samples with spherical tips of 130, 200, 260 and $300 \mu \mathrm{m}$ radii. Therein, it was shown that the Oliver-Pharr approach [31] simply reduces to the Hertz's solution for the case of elastic contact. Furthermore, for Al 6061-T6 [18], the Oliver-Pharr approach [31] yielded excellent results in elastic, elasto-plastic and fully developed plasticity regimes. 
In the present research, the Oliver-Pharr approach [31] was applied on the fused silica sample to obtain contact depth $h_{c}$ and unloading stiffness $S$ of each load-displacement curve, with $S$ and $h_{c}$ determined with

$S=\alpha m\left(h_{\max }-h_{\mathrm{f}}\right)^{m-1}$,

$h_{\mathrm{c}}$

$=h_{\max }$

$-\varepsilon \frac{F_{\max }}{S}$

respectively, where, $\alpha, m$ and $h_{\mathrm{f}}$ in Eq. (1) are parameters obtained by fitting the unloading curve, and $\varepsilon$ in Eq. (2) is a tip dependent constant ( 0.75 for a spherical tip). The effective radius $R_{\text {eff }}$ of a spherical tip is simply given as

$R_{\mathrm{eff}}=\frac{\left(a_{\mathrm{c}}^{2}+h_{c}^{2}\right)}{2 h_{c}}$,

with contact radius $a_{\mathrm{c}}$ given as

$a_{\mathrm{c}}=\frac{S}{2 E_{r}}$.

The reduced elastic modulus $E_{r}$ of the reference sample can be obtained via

$$
\frac{1}{E_{\mathrm{r}}}=\frac{1-v_{\mathrm{s}}^{2}}{E_{\mathrm{s}}}+\frac{1-v_{\mathrm{i}}^{2}}{E_{\mathrm{i}}}
$$

$E_{\mathrm{r}}$ of fused silica in Eq. (5) can be found by utilizing the elastic modulus $E_{\mathrm{i}}$ and Poisson's ratio $v_{\mathrm{i}}$ of the diamond tip (1141 GPa and 0.07, respectively) and the elastic modulus $E_{\mathrm{s}}$ and Poisson's ratio $v_{\mathrm{s}}$ of fused silica (72.1 $\mathrm{GPa}$ and 0.18 , respectively). $R_{\text {eff }}$ is calculated using Eq. (3) by applying the determined values of $h_{\mathrm{c}}$ and $a_{\mathrm{c}}$.

Fig. 2 illustrates $R_{\text {eff }}$ of 3,10 and $50 \mu \mathrm{m}$ radii tips as a function of $h$ where $R_{\text {eff }}$ of 3 and 10 $\mu \mathrm{m}$ tips were found to increase with increasing $h$, eventually reaching a plateau where the 
variations in $R_{\text {eff }}$ were rather insignificant. The range of $h$ within this plateau for the $3 \mu \mathrm{m}$ tip was found to be between 238 and $380 \mathrm{~nm}$, and the range of $h$ for the $10 \mu \mathrm{m}$ tip was between 534 and $1494 \mathrm{~nm}$. In each case, the mean value of the tip radii within this plateau region was considered as the actual radius $R_{\text {act }}$ of the tip. $R_{\text {act }}$ of the $3 \mu \mathrm{m}$ tip ( $R_{\text {act3 }}$ in the following) was found to be $2.12 \mu \mathrm{m}$ and the $R_{\text {act }}$ of the $10 \mu \mathrm{m}$ tip ( $R_{\text {act10 }}$ in the following) was found to be 8.05 $\mu \mathrm{m}$. The magnitudes of maximum error values (standard error of mean) in $R_{\text {eff }}$ for 3,10 and 50 $\mu \mathrm{m}$ radii tips were $0.0343,0.124$ and $0.504 \mu \mathrm{m}$ respectively. Due to the small magnitudes of error in the measurement of $R_{\text {eff }}$, the standard error bars have been omitted in Fig. 2.

As illustrated in Fig. 2, $R_{\text {eff }}$ of the $50 \mu \mathrm{m}$ tip was found to increase with increasing $h$ and reach the load limit of the XP head $(500 \mathrm{mN})$, above which the determination of $R_{\text {eff }}$ is impractical using fused silica as reference sample. Therefore, the $R_{\text {act }}$ for the $50 \mu \mathrm{m}$ radius tip was assumed to be equal to the nominal radius of the tip ( $R_{\text {nom50 }}$ in the following). Similarly, as the $R_{\text {eff }}$ of $250 \mu \mathrm{m}$ radius tip could not be calculated above indentation depth of $650 \mathrm{~nm}$, the $R_{\text {act }}$ was assumed to be equal to the nominal radius of the tip ( $R_{\text {nom } 250}$ in the following $)$.

\subsubsection{Indentation testing on epoxy}

The indentation experiments on epoxy were conducted with the same loading and unloading times (denoted as $T$ in the following). Two different $T$ of 20 and $200 \mathrm{~s}$ with a holding time of $150 \mathrm{~s}$ were considered. A sufficient amount of holding time is needed to stabilize the indentation depth and strain fields in the material so that they do not further change with holding time [32]. The maximum $h$ was limited to $30 \%$ of the nominal tip radius or the depth corresponding to the upper limit of the applied indenter head (whichever limit was reached first). Indentation depths above $30 \%$ tip radius might lead to inaccurate results as the tip might no longer be spherical at these indentation depths [20]. The lower limits of $h$ with the 3 and $10 \mu \mathrm{m}$ tips were chosen to be 
238 and $534 \mathrm{~nm}$, respectively (lower limits of the plateau region, see Fig. 2). However, the lower limits of the indentation depth for the indentations with 50 and $250 \mu \mathrm{m}$ tips were selected to be 480 and $400 \mathrm{~nm}$, respectively. These depths were assumed to be reasonable lower limits to depict the variation in $E$ over the range of $h$.

\section{Results and discussion}

\subsection{Measurement of elastic moduli by spherical indentation testing}

The elastic moduli were determined from the load-displacement curves obtained by indentation testing with different spherical tips. Since significant plastic deformation was observed over the entire range of $h$ with the 3,10 and 50 micron radii tips, the Oliver \& Pharr approach [31] was applied to obtain $E$ of the epoxy sample (assuming a Poisson's ratio of 0.35). However, for the $250 \mu \mathrm{m}$ radius tip, $E$ was obtained using the Hertzian contact theory [30] as the deformations remained elastic and no residual impressions were observed. The applied tip radii for 3 and $10 \mu \mathrm{m}$ tips were $R_{\text {act3 }}$ and $R_{\text {act10, }}$, respectively, whereas nominal tip radii values $R_{\text {nom50 }}$ and $R_{\text {nom } 250}$ were used for the 50 and $250 \mu \mathrm{m}$ tips, respectively.

Fig. 3 depicts $E$ of epoxy with respect to $h$ for different $T$ (20 and $200 \mathrm{~s}$ ). The magnitudes of maximum error values (standard error of mean) in the measurement of $E$ for 3, 10, 50 and 250 $\mu \mathrm{m}$ radii tips for a $T$ of $20 \mathrm{~s}$ were $0.01,0.06,0.06$ and $0.04 \mathrm{GPa}$, respectively. For a $T$ of $200 \mathrm{~s}$, the magnitudes of maximum error values for $3,10,50$ and $250 \mu \mathrm{m}$ radii tips were $0.02,0.03$, 0.06 and $0.06 \mathrm{GPa}$, respectively. As before, due to the negligible errors in the measurement of $E$, the standard error bars have been omitted in Fig. 3 and subsequent figures.

As illustrated in Fig. 3, $E$ obtained with 3 and $10 \mu \mathrm{m}$ radii tips for different $T$ remain essentially constant with respect to $h$, while sharp decreases in the measured $E$ were obtained with 50 and $250 \mu \mathrm{m}$ radii tips at $h$ below $1.6 \mu \mathrm{m}$ and $600 \mathrm{~nm}$, respectively. These sharp 
decreases in $E$ could be attributed to deviations in $R_{\text {eff }}$ from $R_{\text {nom }}$ of tips at these depths. The determination of $E$ based on the Oliver and Pharr approach [31] is dependent on the accurate determination of the contact area $A_{\mathrm{C}}$. The contact area $A_{\mathrm{C}}$ is related to $R_{\text {eff }}$ and contact depth $h_{\mathrm{c}}$ via

$A_{\mathrm{C}}=\pi\left(2 h_{\mathrm{c}} R_{\mathrm{eff}}-h_{\mathrm{c}}^{2}\right)$.

Therefore, deviations of $R_{\text {eff }}$ from $R_{\text {nom }}$ can affect the determination of $E$ significantly.

Similar influences of geometrical imperfections of spherical tips on the determination of the material properties have been reported by various researchers $[17,19,21,27]$. However, using $R_{\text {nom }}$ of four different tips (130, 200, 260 and $\left.300 \mu \mathrm{m}\right)$ and the Oliver-Pharr approach, Herbert et al.[18] were able to predict the elastic moduli of fused silica and Al 6061-T6 with reasonable accuracy. However, the radii of the tips used in their work were significantly larger than three (3, 10 and $50 \mu \mathrm{m}$ ) of the four tips used in this work. Since the manufacturing complexity reduces with increasing tip radii [27], the influence of tip imperfections should be relatively far less pronounced for tips with higher $R_{\text {nom }}$ (e.g., $250 \mu \mathrm{m}$ ) compared to tips with smaller $R_{\text {nom }}$ (e.g., the 3 and $10 \mu \mathrm{m}$ tips). As a consequence, at lower $h$, the discrepancy between $R_{\text {eff }}$ and $R_{\text {nom }}$ for tips with higher $R_{\text {nom }}$ should be significantly less compared to tips of smaller $R_{\text {nom }}$, resulting in reasonably accurate $E$ even at lower $h$, as obtained by Herbert et al [18]. However, as $R_{\text {act }}$ (instead of $R_{\text {nom }}$ ) of the 3 and $10 \mu \mathrm{m}$ radii tips has been used to obtain $E$, the imperfections of these tips should have a negligible influence on the experimental results at lower $h$ (see Fig. 3).

The results depicted in Fig. 3 indicate an increase in $E$ with reduction in the tip radius. Also, as evident in Fig. 3, with the increase in $T$ from 20 to $200 \mathrm{~s}$ (with the holding time being kept constant at $150 \mathrm{~s}$ ), there is a marked decrease in $E$ obtained with all the spherical tips. As the deformation in polymers is known to be rate dependent, with the increase in $T$, the measured $E$ is 
expected to be influenced by rearrangement and relaxation of polymer chains. A similar influence of $T$ on the measured $E$ has been previously observed with spherical indentation testing on PDMS [7].

To further illustrate the abovementioned results, $E$ is plotted with respect to tip radius in Fig. 4. Therein, the $E$ values obtained with 50 and $250 \mu \mathrm{m}$ tips at $h$ where the tip imperfection was found to have a significant influence (data below $1.6 \mu \mathrm{m}$ and $600 \mathrm{~nm}$ indentation depths for 50 and $250 \mu \mathrm{m}$ tips, respectively), have been neglected. As evident in Fig. 4, the measured $E$ is dependent on the radius of the spherical indenter tip. The influence of surface effects, adhesion and second order displacement gradients as some of the possible explanations for such a dependence of $E$ on the tip radius is discussed below.

\subsection{Possible explanations for the dependence of measured $E$ on the tip radius}

\subsubsection{Surface effects}

It is well known from various indentation studies [33-35] that rough surfaces can adversely influence nanoindentation results, especially at shallow indentation depths. To assess the influence of surface roughness on the measured $E$, two different areas $(217.429 \mu \mathrm{m}$ x $290 \mu \mathrm{m})$ of the epoxy sample were analyzed using Keyence VK-X series 3D laser scanning confocal microscope (Elmwood Park, NJ). Fig. 5 depicts the multi-line surface roughness profile obtained by scanning multiple lines on one of the sample areas. The average surface roughness $R_{\mathrm{a}}$ was found to be $13 \mathrm{~nm}$ which is an order of magnitude lower than the minimum indentation depth $(315 \mathrm{~nm})$ of the presented indentation experiments. Therefore, one may assume that the influence of surface roughness on the measured $E$ was negligible.

It was also assumed that the tested epoxy is homogeneous and that the material properties do not change with $h$. However, at distances of less than $100 \mathrm{~nm}$ from free surfaces some changes 
of the polymer properties could be present [36]. Furthermore, particle embedment experiments conducted by Taskin and McKenna [37] revealed that the surface of poly( $\alpha$-methylstyrene) was found to be softer than the macroscopic material at ambient temperatures. However, the changes in $E$ in the present research were found well beyond $100 \mathrm{~nm}$ (see Fig. 3) where the surface softening effects should be negligible.

\subsubsection{Adhesion}

The nanoindentation experiments could be significantly influenced by adhesive forces between the tip and the sample $[38,39]$. The Hertz theory [30] and the Oliver and Pharr approach [31] (also based on the Hertz theory) used here to determine $E$, assume negligible adhesion between the sample and the tip [39]. If significant adhesive forces exist between the tip and the sample, the analysis using the Hertz theory [30] leads to underestimation of the contact area and the effective load experienced by the material, which would result in an overestimation of the elastic modulus [39]. Therefore, neglecting the influence of adhesion may lead to significant errors in determination of $E$. During the loading sequence of the indentation procedure, if adhesive forces are significant, the initial contact point between the tip and the sample could be different compared to the initial contact point detected by the indentation system. To determine the influence of adhesion during loading, sufficient data has to be collected before the point of contact between the tip and the sample [38]. Similarly, as the tip is withdrawn from the sample at the end of the unloading sequence, adhesion is indicated by a negative load (pull-off force). Therefore, to determine the influence of adhesion during unloading, the data has to be collected to zero displacement (i.e., until the total separation of the tip from the sample surface) [38]. Fig. 6 depicts the raw load/raw displacement data corresponding to lowest $h$ obtained with the $50 \mu \mathrm{m}$ tip. Therein, the load displacement data has been collected before the point of contact between 
the tip and the sample, and the unloading data has been obtained until zero displacement. As evident in Fig. 6, there is a negligible influence of adhesion during the loading and unloading sequences of the indentation procedure.

\subsubsection{The influence of second order displacement gradients}

As surface effects and adhesion were found to have negligible influence on the measurement of $E$, the influence of second order displacement gradients (such as strain and rotation gradients) as a possible explanation for such a dependence of $E$ on the tip radius is examined here. The influence of second order displacement gradients (denoted as $\chi$ in the following) on the indentation testing of polymers with spherical and Berkovich tips has been previously elucidated in $[7,8,10,11]$. However, for the convenience of the reader, a brief review is provided below followed by a corresponding interpretation of the here obtained results.

Experimental studies conducted on metals at micron to submicron scales have revealed that the length scale dependent deformation can be attributed to geometrically necessary dislocations which can be related to strain gradients in plastic deformation [40,41]. However, the literature available on the origin of length scale dependent elasto-plastic deformation of glassy polymers is rather sparse. Based on a molecular theory of yielding for glassy polymers, a strain gradient plasticity law and a related indentation model was developed by Lam et al [42]. This indentation model has been used by Chong et al. [43] and Swaddiwudhipong et al. [44] to capture the length scale dependent deformation of glassy polymers.

While the abovementioned study by Lam et al. [42] considers the length scale dependent deformation in polymers to be associated with plastic deformation, the micro-cantilever beam bending experiments conducted on epoxy and polypropylene by Lam et. al. [45] and McFarland et al. [46], respectively, exhibited significant increases in beam stiffness with decreasing 
thickness in elastic deformation. Furthermore, indentation experiments by Alisafaei et al. [11] revealed that $E$ of epoxy obtained by indentation tests using a Berkovich tip and applying Oliver and Pharr approach (based on the evaluation of the elastic unloading sequence) increased with decreasing $h$. While for polymers such increases in $E$ can be attributed to significant elastic nature of length scale dependent deformation [11], similar changes in $E$ (with decreasing $h$ ) have not been observed in metals as the length scale dependent deformation in metals is not associated with elastic deformation.

While Alisafaei at al. [11] found that $E$ of epoxy determined with a Berkovich tip increased with decreasing $h, E$ obtained by spherical indentation with tips of different nominal radii (see Fig. 3) in this work remains essentially independent of $h$ (neglecting $E$ obtained with 50 and 250 $\mu \mathrm{m}$ tips at lower $h$ where tip imperfection was found to have a significant influence). Also, as evident in Fig. 4, $E$ obtained with tips of different nominal radii increase with decreasing tip radius $R$. This dependence of $E$ on the tip geometry (Berkovich or spherical) is examined below.

The length scale dependent deformation in polymers can be described based on increases in $\chi$ with changing length scale [14-16,42-45]. As the length scale dependent deformation in polymers (as mentioned above) is dependent on tip geometry, a relation between tip geometry and $\chi$ is examined here. The relations between $\chi$, and conical (Berkovich, Vickers, etc.) and spherical indenter tips are found in [1,47] and are illustrated in Fig. 7. As seen therein, while $\chi$ increases with decreasing $h$ with conical indentation, $\chi$ is essentially independent of $h$, but inversely proportional to the tip radius $R$ with spherical indentation testing. As increases in $\chi$ (with changing length scale) describe the length scale dependent deformation in polymers, increases in $\chi$ with decreasing $R$ should correspondingly influence the measurement of $E$ with spherical indentation testing. Accordingly, as the length scale dependent deformation in 
polymers is present in elastic deformation, $E$ obtained by spherical indentation is expected to increase with decreasing $R$ due to a corresponding increase in $\chi$. As mentioned above, such an increase in $E$ with decreasing $R$ is clearly evident in Fig. 4.

While the existing strain gradient elasticity theories can predict the length scale dependent deformation, these theories are phenomenological and lack a micromechanical motivation for polymers. On a molecular scale, as non-local molecular interactions are prominent [48], a micromechanically motivated model describing the length scale dependent deformation should be able to incorporate the effects of such molecular interactions. The non-local higher order gradient model developed by Nikolov et al. [15] can be micromechanically motivated by relating increases in $\chi$ and corresponding length scale dependent deformation to changes in non-local potential energy, which is similar to Frank energy [49] (which originates from the rotational entropy of polymer chains and the molecular interaction forces) of liquid crystal polymers.

With $\chi$ scaling with $1 / h$ for a Berkovich tip and $1 / R$ for a spherical tip (see Fig. 7 ), $E$ measured with a Berkovich tip at large, macroscopic $h$ would predict the same value as with a spherical tip of large curvature radius. Fig. 8 depicts $E$ with the Berkovich tip at different $T$ (5, 20 and $200 \mathrm{~s}$ ) and $E$ obtained with the $250 \mu \mathrm{m}$ spherical tip at two different $T$ (20 and $200 \mathrm{~s}$ ). Therein, it can be seen that there are some differences in $E$ obtained with the Berkovich tip at larger $h$ and $E$ obtained with the $250 \mu \mathrm{m}$ spherical tip. Similar differences in $E$ were also observed in natural rubber [8] where the Berkovich tip at macroscopic $h$ yielded higher $E$ compared to $E$ obtained with a spherical tip of $250 \mu \mathrm{m}$ radius. In this respect, it is worth noting that the Berkovich tip has 3 rather sharp edges which results in the presence of strong $\chi$ around the edges and correspondingly higher stiffness, even at higher macroscopic $h$ which are absent in spherical tips. Stiffening effects have been found in electro-polished nickel which has been 
analyzed with strain gradient finite element simulations [50]. Therein, indentations with a Berkovich tip required higher forces to penetrate a sample to a given depth than with a conical tip. This was attributed to substantially higher strain gradients in the vicinity of the edges of the Berkovich tip which are absent on the smooth surface of a conical tip. Following this notion, in addition to the dependence of $E$ on $\chi, E$ measured with the Berkovich tip is also dependent on $\chi$ at the edges.

Taking into account that the surface of a spherical tip is significantly smoother in comparison with a conical tip, the measured $E$ with a spherical tip should be lower than that obtained with a conical tip and much lower than that obtained with a Berkovich tip at macroscopic $h$. Following this notion, the differences in $E$ measured with the spherical tip of $250 \mu \mathrm{m}$ nominal radius and the Berkovich tip at macroscopic $h$ (see Fig. 8) can be attributed to a certain extent to the additional influence of $\chi$ at the edges of the Berkovich tip. However, as $T$ is increased from 5 to 200 s, $E$ obtained at macroscopic $h$ (with $T=200$ s) with Berkovich tip (see Fig. 8) is closer to that obtained with the spherical tip of $250 \mu \mathrm{m}$ radius. Considering that polymers are strongly rate dependent, such decreases in $E$ with increasing $T$ is not surprising. However, even with the largest $T(200 \mathrm{~s})$, the influence of $\chi$ at the edges of the Berkovich tip could still result in a macroscopic $E$ higher than that obtained with the spherical tip of $250 \mu$ m radius.

\section{Conclusions}

Indentation testing was performed on epoxy with spherical tips of four different radii to examine the dependence of elastic moduli measurements on the spherical indenter tip radius. Tip imperfection studies conducted on spherical tips of 3 and $10 \mu \mathrm{m}$ radii, revealed significant variations in the effective tip radius from its nominal value, especially at small indentation depths. Taking these tip imperfections into account, a significant increase in the elastic moduli 
was observed with decreasing tip radius. The observed increase in the measured elastic moduli with decreasing tip radius is attributed to increase in second order displacement gradients with decreasing tip radius. This notion is consistent with indentation results obtained by applying Berkovich tips where significant increases in the elastic moduli were observed with decreasing indentation depth, which can also be related to the influence of second order displacement gradients.

\section{Acknowledgments}

The material of this article is based on work supported by the U.S. National Science Foundation under Grants No. 1102764 and No. 1126860. The authors would also like to acknowledge Mr. Christian Skipper from the Keyence Corporation of America for the assistance provided in the surface roughness measurements.

\section{References}

[1] J. G. Swadener, E. P. George, G. M. Pharr, The correlation of the indentation size effect measured with indenters of various shapes, J. Mech. Phys. Solids 50 (2002) 681—694.

[2] W. W. Gerberich, N. I. Tymiak, J. C. Grunlan, M. F. Horstemeyer, M. I. Baskes, Interpretations of Indentation Size Effects, J. Appl. Mech. 69 (2002) 433—442.

[3] T. Jin, X. Niu, G. Xiao, Z. Wang, Z. Zhou, G. Yuan, X. Shu, Effects of experimental variables on PMMA nano-indentation measurements, Polym. Test. 41 (2015) 1-6.

[4] G. L. Oliveira , C. A. Costa , S. C. S. Teixeira , M. F. Costa, The use of nano- and microinstrumented indentation tests to evaluate viscoelastic behavior of poly(vinylidene fluoride) (PVDF), Polym. Test. 34 (2014) 10—16. 
[5] A. Karimzadeh, M. R. Ayatollahi, Investigation of mechanical and tribological properties of bone cement by nano-indentation and nano-scratch experiment, Polym. Test. 31 (2012) 828833.

[6] F. Alisafaei, C.-S. Han, S. H. R. Sanei, On the time and indentation depth dependence of hardness, dissipation and stiffness in polydimethylsiloxane, Polym. Test. 32 (2013)12201228.

[7] C.-S. Han, S. H. R. Sanei, F. Alisafaei, On the origin of indentation size effects and depth dependent mechanical properties of elastic polymers, J. Polym. Eng. 36 (2015) 103-111.

[8] G. Chandrashekar, F. Alisafaei, C.-S. Han, Length scale dependent deformation in natural rubber, J. Appl. Polym. Sci. 132 (2015) 42683.

[9] D. C. C. Lam, A. C. M. Chong, Effect of cross-link density on strain gradient plasticity in epoxy, Mater. Sci. Eng. A 281 (2000) 156-161.

[10] F. Alisafaei, C.-S. Han, N. Lakhera, Characterization of indentation size effects in epoxy, Polym. Test. 40 (2014) 70-78.

[11] F. Alisafaei, C.-S. Han, N. Garg, On couple-stress elasto-plastic constitutive frameworks for glassy polymers, Int. J. Plast. 77 (2016) 30—53.

[12] L. Malekmotiei, A. S. Dooki, G. Z. Voyiadjis, Nanoindentation Study of Yielding and Plasticity of Poly(methylmethacrylate), Macromolecules 48 (2015) 5348—5357.

[13] F. Alisafaei, C.-S. Han, Indentation depth dependent mechanical behavior in polymers, Adv. Cond. Matt. Phys. 2015 (2015) 391579, 20 pages.

[14] C.-S. Han, S. Nikolov, Indentation size effects in polymers and related rotation gradients, J. Mater. Res. 22 (2007) 1662-1672. 
[15] S. Nikolov, C.-S. Han, D. Raabe, On the origin of size effects in small-strain elasticity of solid polymers, Int. J. Sol. Struc. 44 (2007) 1582-1592.

[16] C.-S. Han, Influence of the molecular structure on indentation size effect in polymers, Mat. Sci. Eng. A 527 (2010) 619—624.

[17] J. Nohava, J. Mencik, A contribution to understanding of low-load spherical IndentationComparison of tests on polymers and fused silica, J. Mater. Res. 27 (2012) 239-244.

[18] E. G. Herbert, G. M. Pharr, W. C. Oliver, B. N. Lucas, J. L. Hay, On the measurement of stress-strain curves by spherical indentation, Thin Solid Films 398 -399 (2001) 331-335.

[19] S.-K. Kang, J.-Y. Kim, I. Kang, D. Kwon, Effective indenter radius and frame compliance in instrumented indentation testing using a spherical indenter, J. Mater. Res. 24 (2009) $2965-2973$.

[20] S. Basu, A. Moseson, M. W. Barsoum, On the determination of spherical nanoindentation stress-strain curves, J. Mater. Res. 21 (2006) 2628-2637.

[21] J. Martegoutte, C. Seguineau, C. Malhaire, T. Fourcade, J.-M. Desmarres, X. Lafonten, P. Francis, Nanoindentation experiments with small tip radii: an experimental method, Des. Test Integ. Pack. MEMS/MOEMS (2010) 225 -230.

[22] J. S. Field, M. V. Swain, A simple predictive model for spherical indentation, J. Mater. Res. 8 (1993) 297-306.

[23] J.-Y. Kim, K.-W. Lee, J.-S. Lee, D. Kwon, Determination of tensile properties by instrumented indentation technique: Representative stress and strain approach, Surf. Coat. Tech. 201 (2006) 4278-4283.

[24] J.-H. Ahn, D. Kwon, Derivation of plastic stress-strain relationship from ball indentations: Examination of strain definition and pileup effect, J. Mater. Res. 16 (2001) 3170-3178. 
[25] W. Yan, Q. Sun, X.-Q. Feng, L. Qian, Analysis of spherical indentation of superelastic shape memory alloys, Int. J. Sol. Struc. 44 (2007) 1-17.

[26] J. G. Swadener, B. Taljat, G. M. Pharr, Measurement of residual stress by load and depth sensing indentation with spherical indenters, J. Mater. Res. 16 (2001) 2091-2102.

[27] G. Constantinides, E. C. C. M. Silva, G. S. Blackman, K. J. Van Vliet, Dealing with imperfection: quantifying potential length scale artefacts from nominally spherical indenter probes, Nanotech. 18 (2007) 305503, 14 pages.

[28] M. L. Oyen, Spherical indentation creep following ramp loading, J. Mater. Res. 20 (2005) $2094-2100$.

[29] R. Seltzer, J.-K. Kim, Y.-W. Mai, Elevated temperature nanoindentation behaviour of polyamide 6, Polym. Int. 60 (2011) 1753-1761.

[30] K. L. Johnson, Contact Mechanics, Cambridge University Press, 1987, p 90.

[31] W. C. Oliver, G. M. Pharr, An improved technique for determining hardness and elastic modulus using load and displacement sensing indentation experiments, J. Mater. Res. 7 (1992) $1564-1583$.

[32] L. Cheng, X. Xia, L. E. Scriven, W. W. Gerberich, Spherical-tip indentation of viscoelastic material, Mech. Mater. 37 (2005) 213-226.

[33] T.-Y. Zhang, W.-H. Xu, M.-H. Zhao, The role of plastic deformation of rough surfaces in the size-dependent hardness, Acta Mat. 52 (2004) 57—68.

[34] D. Hoffman, I. Miskioglu, K. E. Aifantis, J. Drelich, Measuring the Surface and Bulk Modulus of Polished Polymers with AFM and Nanoindentation, J. Adh. Sci. Technol. 26 (2012) 1201-1220. 
[35] E. Donenelly, S. P. Baker, A. L. Boskey, M. C. H. van der Muelen, Effects of surface roughness and maximum load on the mechanical properties of cancellous bone measured by nanoindentation, J. Biomed. Mater. Res. A 77 (2006) 426—435.

[36] R. D. Priestley, C. J. Ellison, L. J. Broadbelt, J. M. Torkelson, Science 309 (2005) 456459.

[37] T. B. Karim, G. B. McKenna, Unusual Surface Mechanical Properties of Poly( $\alpha$ methylstyrene): Surface Softening and Stiffening at Different Temperatures, Macromolecules 45 (2012) 9697-9706.

[38] J. C. Kohn, D. M. Ebenstein, Eliminating adhesion errors in nanoindentation of compliant polymers and hydrogels, J. Mech. Beh. Bio. Mat. 20 (2013) 316-326.

[39] D. M. Ebenstein, K. J. Wahl, A comparison of JKR-based methods to analyze quasi-static and dynamic indentation force curves, J. Coll. Inter. Sci. 298 (2006) 652-662.

[40] N. A. Fleck, G. M. Muller, M. F. Ashby, J. W. Hutchinson, Strain gradient plasticity: theory and experiment, Acta Metall. Mater. 42 (1994) 475-487.

[41] W. D. Nix, H. Gao, Indentation size effects in crystalline materials: a law for strain gradient plasticity, J. Mech. Phys. Sol. 46 (1998) 411—425.

[42] D. C. C. Lam, A. C. M. Chong, Indentation model and strain gradient plasticity law for glassy polymers, J. Mater. Res. 14 (1999) 3784-3788.

[43] A. C. M. Chong, D. C. C. Lam, Strain gradient plasticity effect in indentation hardness of polymers, J. Mater. Res. 14 (1999) 4103-4110.

[44] S. Swaddiwudhipong, L. H. Poh, J. Hua, Z. S. Liu, K. K. Tho, Modeling nano-indentation tests of glassy polymers using finite elements with strain gradient plasticity, Mater. Sci. Eng. A 404 (2005) 179-187. 
[45] D. C. C. Lam, F. Yang, A. C. M. Chong, J. Wang, P. Tong, Experiments and theory in strain gradient elasticity, J. Mech. Phys. Sol. 51 (2003) 1477-1508.

[46] A. W. McFarland, J. S. Colton, Role of material microstructure in plate stiffness with relevance to microcantilever sensors, J. Micromech. Microeng. 15 (2005) 1060—1067.

[47] N. I. Tymiak, D. E. Kramer, D. F. Bahr, T. J. Wyrobek, W. W. Gerberich, Plastic strain and strain gradients at very small indentation depths, Acta Mat. 49 (2001) 1021—1034.

[48] S. Ghosh, A. Kumar, V. Sundararaghavan, A. M. Waas, Non-local modeling of epoxy using an atomistically-informed kernel, Int. Journ. Sol. Struc. 50 (2013) 2837-2845.

[49] P. G. de Gennes, J. Prost, The Physics of Liquid Crystals, Oxford University Press, 1993.

[50] S. Swaddiwudhipong, K. K. Thao, J. Hua, Z. S. Liu, Mechanism-based strain gradient plasticity in $\mathrm{C}^{0}$ axisymmetric element, Int. Journ. Sol. Struc. 43 (2006) 1117-1130. 
Figure captions:

1. Fig. 1. Schematic load-displacement curve

2. Fig. 2. $R_{\text {eff }}$ versus indentation depth, $h$, for spherical tips of different nominal radii

3. Fig. 3. Elastic modulus versus indentation depth for spherical tips of different radii

4. Fig. 4. Elastic modulus versus tip radius

5. Fig. 5. (A) Sample area (B) Multi line roughness profile

6. Fig. 6. Raw load versus raw displacement corresponding to minimum $F_{\text {max }}$ (lowest indentation depth) obtained with the $50 \mu \mathrm{m}$ spherical tip

7. Fig. 7. $\chi$ related to (A) conical and (B) spherical tips

8. Fig. 8. Comparison of elastic moduli determined with Berkovich and spherical tips 


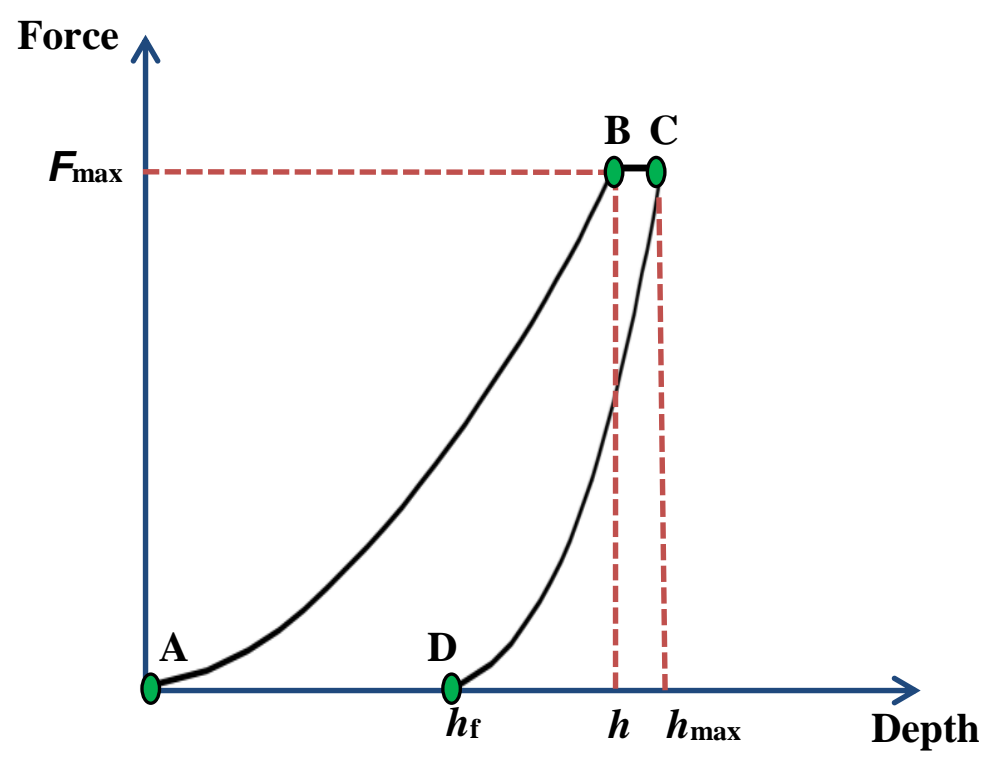

Fig. 1. Schematic load-displacement curve 


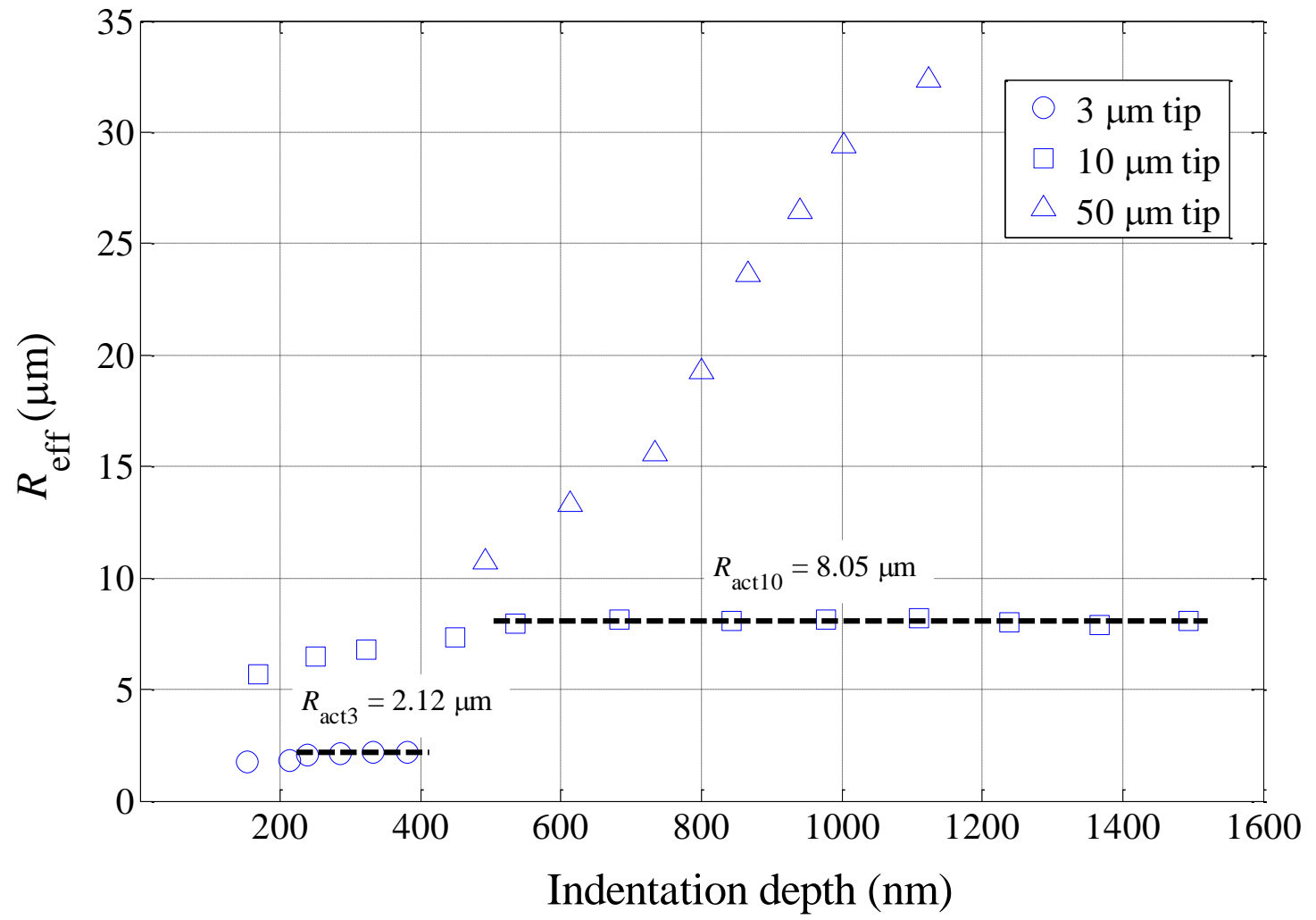

Fig. 2. $R_{\text {eff }}$ versus indentation depth, $h$, for spherical tips of different nominal radii 


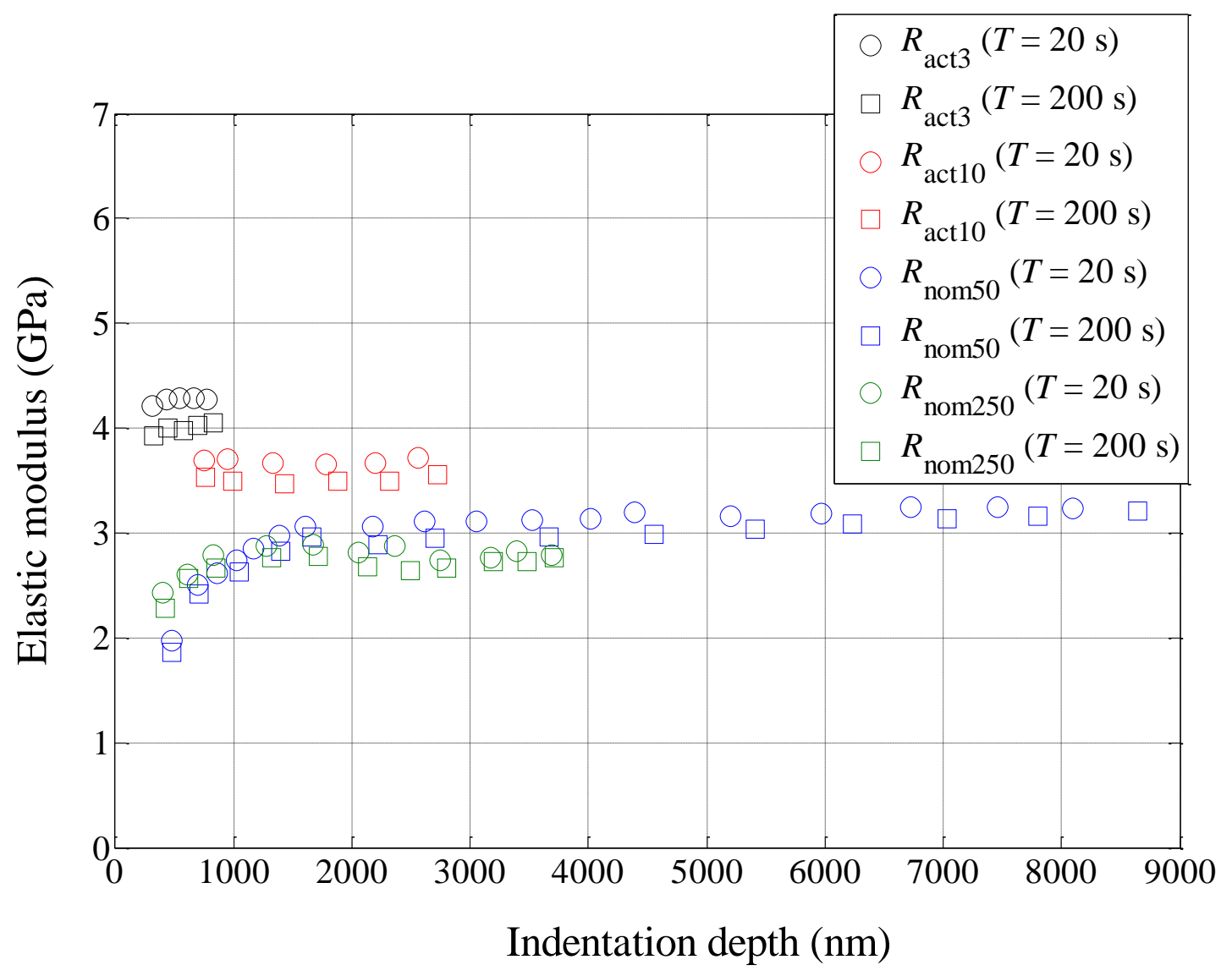

Fig. 3. Elastic modulus versus indentation depth for spherical tips of different radii 


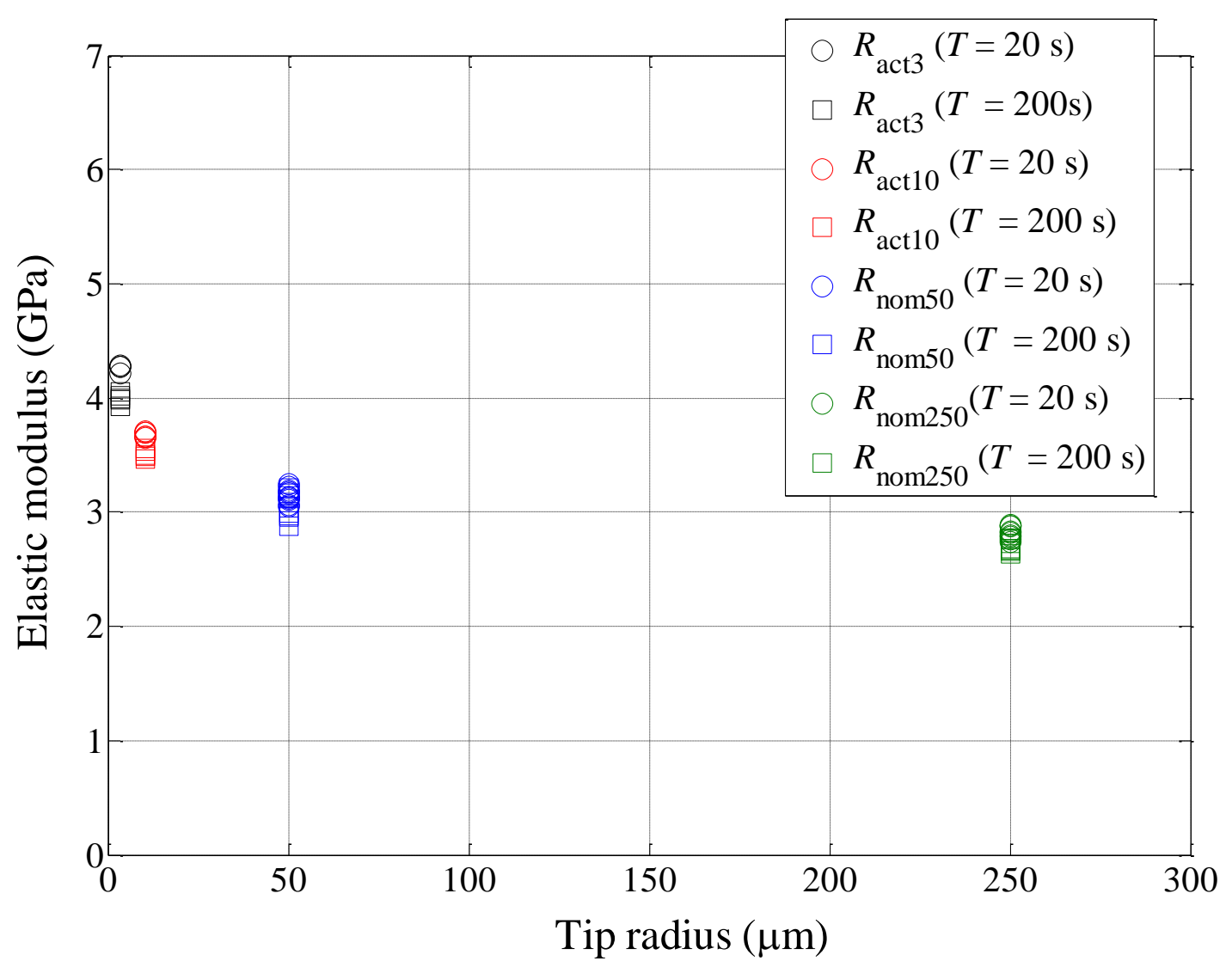

Fig. 4. Elastic modulus versus tip radius 

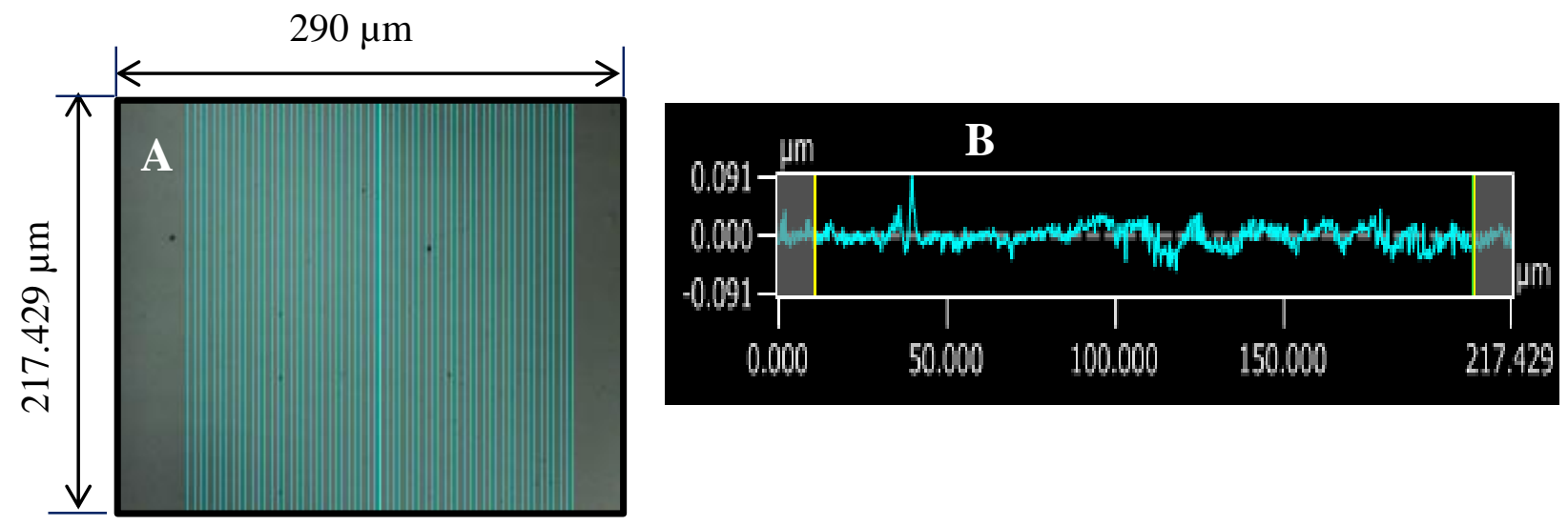

Fig. 5. (A) Sample area (B) Multi line roughness profile 


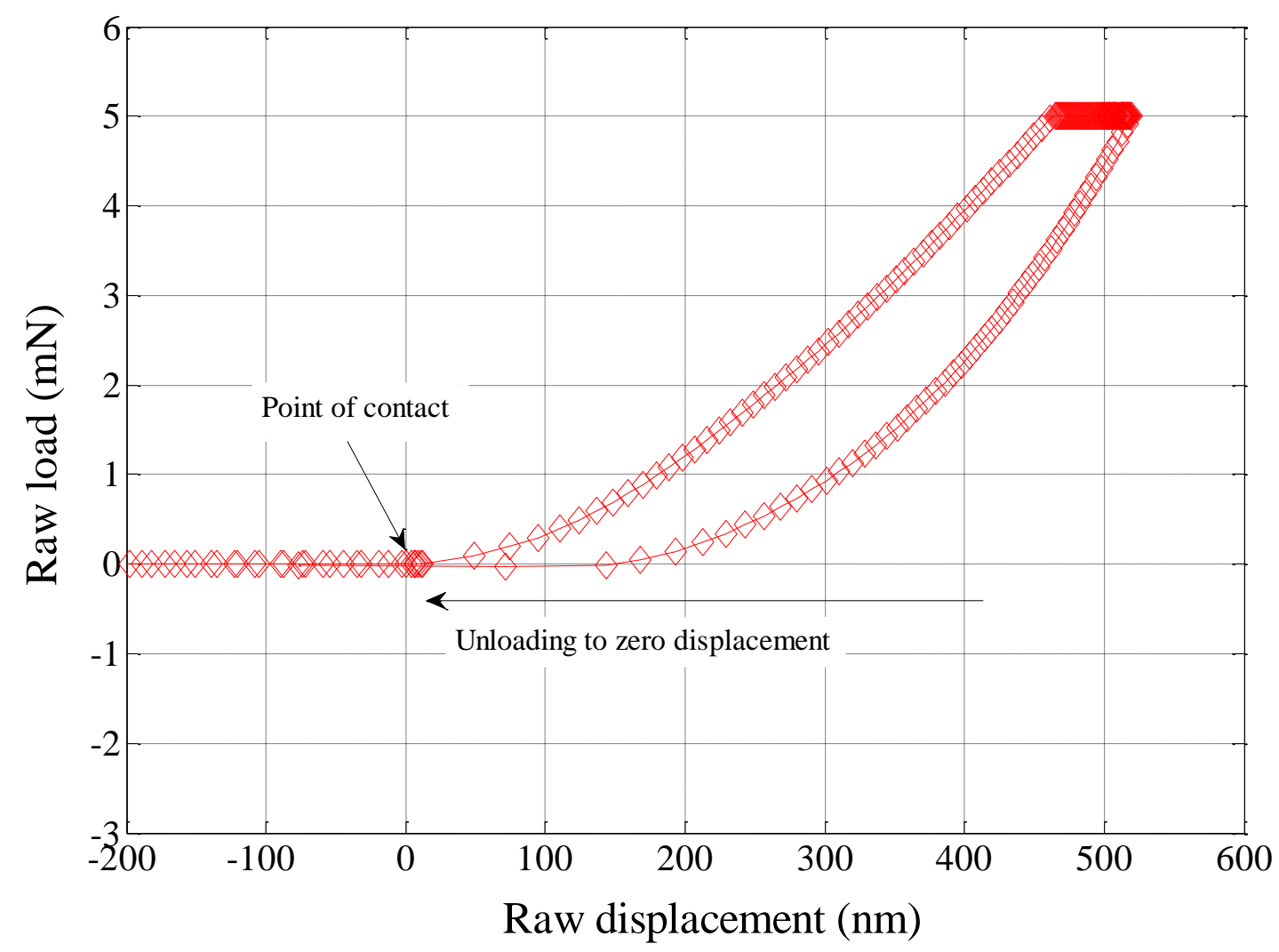

Fig. 6. Raw load versus raw displacement corresponding to minimum $F_{\max }$ (lowest indentation depth) obtained with the $50 \mu \mathrm{m}$ spherical tip 


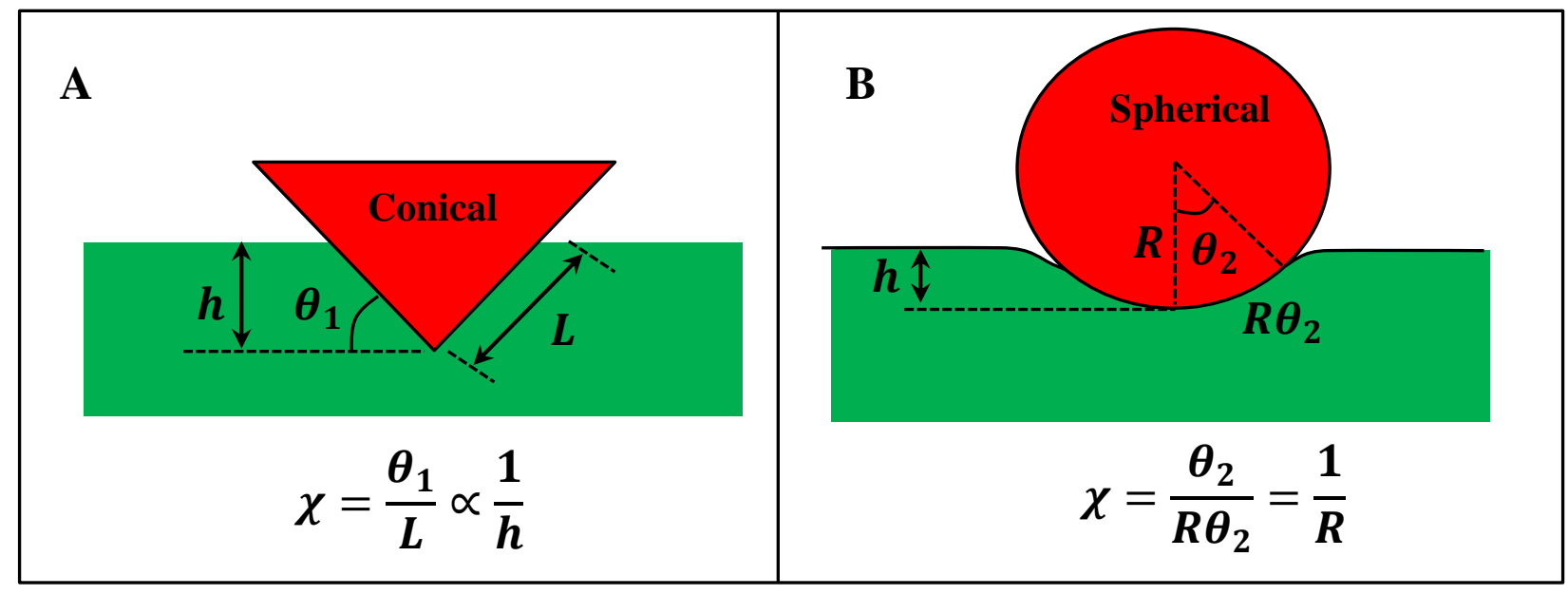

Fig. 7. $\chi$ related to (A) conical and (B) spherical tips 


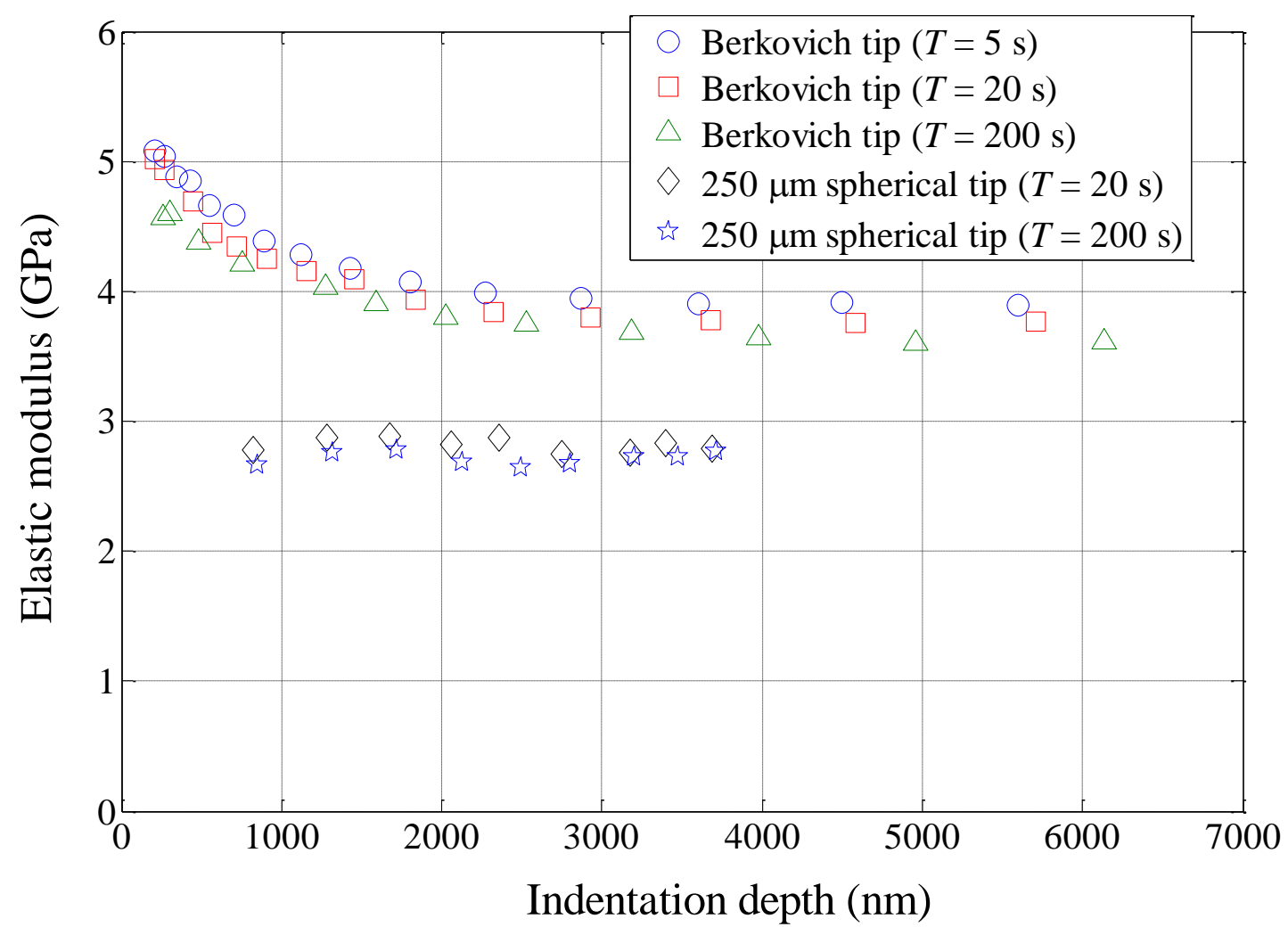

Fig. 8. Comparison of elastic moduli determined with Berkovich and spherical tips 